\title{
First results of Mercator observations of variable B stars
}

\section{P. De Cat ${ }^{1,6}$, J. De Ridder ${ }^{1,6}$, K. Uytterhoeven ${ }^{1,2}$, G. Davignon ${ }^{1,2}$,} G. Raskin ${ }^{1,2}$, J. Cuypers ${ }^{3}$, C. Schoenaers ${ }^{3}$, J. Daszyńska-Daszkiewicz ${ }^{1,4}$, C. Aerts ${ }^{1}$, H. Van Winckel ${ }^{1}$, M. Ausseloos ${ }^{1}$, E. Broeders ${ }^{1}$, W. De Meester ${ }^{1}$, J. Vanautgaerden ${ }^{1}$, R. Van Malderen ${ }^{1}$, B. Vandenbussche ${ }^{1}$, B. Acke ${ }^{1}$, G. Decin ${ }^{1}$, L. Decin ${ }^{1}$, K. Kolenberg ${ }^{1}$, T. Maas ${ }^{1}$, S. De Ruyter ${ }^{1,5}$, M. Reyniers ${ }^{1}$, T. Reyniers ${ }^{1}$, C. Van Kerckhoven ${ }^{1}$, C. Waelkens ${ }^{1}$

\section{${ }^{1}$ Instituut voor Sterrenkunde, Katholieke Universiteit Leuven,} Celestijnenlaan 200 B, B-3001 Leuven, Belgium

${ }^{2}$ Mercator Telescope, Calle Alvarez de Abreu 70, E-38700 Santa Cruz de La Palma, Spain

${ }^{3}$ Koninklijke Sterrenwacht van België, Ringlaan 3, B-1180 Brussel, Belgium

${ }^{4}$ Astromical Institute of the Wroclaw University, ul. Kopernika 11, 51-622 Wroclaw, Poland

${ }^{5}$ Sterrenkundig Observatorium, Universiteit Gent, Krijgslaan 281-S9, 9000 Gent, Belgium

\begin{abstract}
We report on the first results from observations of 28 variable B stars obtained with the new Mercator telescope (La Palma). Besides confirming the pulsational nature of known and candidate $\beta$ Cephei and slowly pulsating $B$ stars, we also present new candidate ellipsoidal variables and spotted stars.
\end{abstract}

\section{Introduction}

The Mercator telescope is a new 1.2-m telescope located on the Roque de los Muchachos observatory on La Palma, Spain. Scientific observations started in northern spring 2001. Currently, the instrument attached to the telescope is "P7" which is a two channel (star + sky) photometer for quasi-simultaneous 7band measurements in the Geneva photometric system. Since 2001 the telescope has been intensively used to observe variable $\mathrm{B}, \mathrm{A}$, and $\mathrm{F}$ main sequence stars. Here we present the data sets obtained for 28 B stars (see Fig. 1), containing both bonafide and candidate $\beta$ Cephei stars and slowly pulsating B stars (SPBs). In the twin paper "First results of Mercator observations of variable A and F

\footnotetext{
${ }^{6}$ Postdoctoral Fellow of the Fund for Scientific Research, Flanders
} 


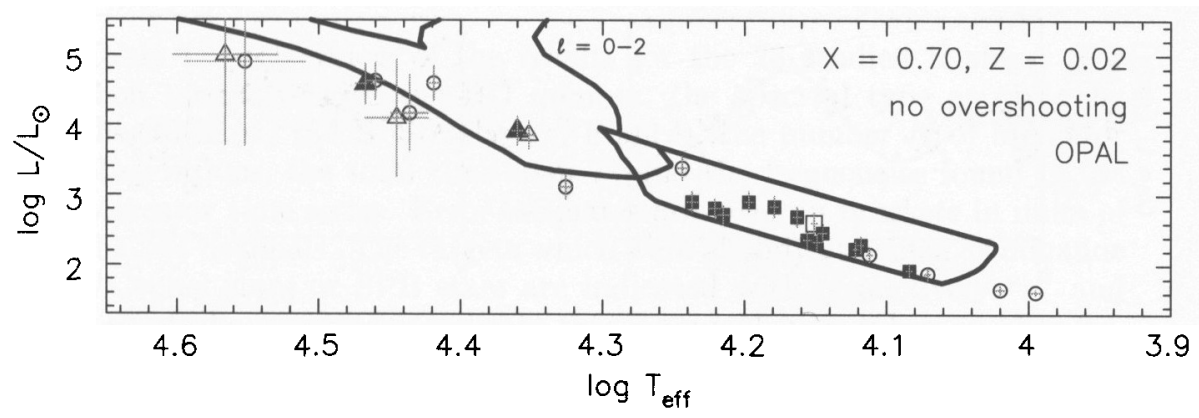

Figure 1. The 28 target stars in the HR diagram. The $\beta$ Cep stars, the SPB stars and the other stars are respectively given as triangles, squares and circles. The objects given with full symbols are selected for long term monitoring with the Mercator telescope (instability strips from Pamyatnykh 1999).

stars" (De Ridder et al., these proceedings), the data sets of 31 of the observed $\mathrm{A}$ and $\mathrm{F}$ stars are presented.

The obtained Mercator time series of the $28 \mathrm{~B}$ stars were subjected to an extensive frequency analysis, where objectivity was imposed by having each star independently analyzed by two or three astronomers. The frequency analysis was done for all the Geneva passbands and colours with both the PDM (Stellingwerf 1978) and Lomb-Scargle (Scargle 1982) method. Our ground-based data-sets suffer from strong aliasing. Therefore, we complemented our frequency search with a (new) analysis of the space-based observations of the Hipparcos satellite in the $H_{p}$ passband. The results for targets of different types of stars are given in the following section. A global overview of the results is given in Table 1.

\section{The data: $\beta$ Cep stars, SPB stars and other stars}

When taking the errors on $\log \left(T_{\text {eff }}\right)$ and $\log \left(L / L_{\odot}\right)$ into account, 9 of the 28 studied objects are situated within the instability strip of the $\beta$ Cep stars. For 5 of them the observed variations in the Geneva passbands and colours are compatible with the expected $\beta$ Cep character (triangles in Fig. 1). They were all already known as $\beta$ Cep stars.

When taking the errors on $\log \left(T_{\text {eff }}\right)$ and $\log \left(L / L_{\odot}\right)$ into account, 16 of the 28 studied objects are situated within the instability strip of the SPBs. For 13 of them the observed variations in the Geneva passbands and colours are compatible with the expected SPB character (squares in Fig. 1). Only 7 of them were previously classified as SPBs.

Even when taking the errors on $\log \left(T_{\text {eff }}\right)$ and $\log \left(L / L_{\odot}\right)$ into account, 3 of the 28 studied objects fall outside the instability strip of both the $\beta$ Cep stars and the SPBs. For these 3 and 5 of the other targets, the observed variations in the Geneva passbands and colours point towards either spotted stars ( $\nu_{\text {rot }}$ and its harmonics; unequal light maxima; main frequency present in colors; e.g. HD 154689 in Fig. 2 ), or ellipsoidal variations ( $\nu_{\text {orb }}$ and $2 \nu_{\text {orb }}$; equal light max- 

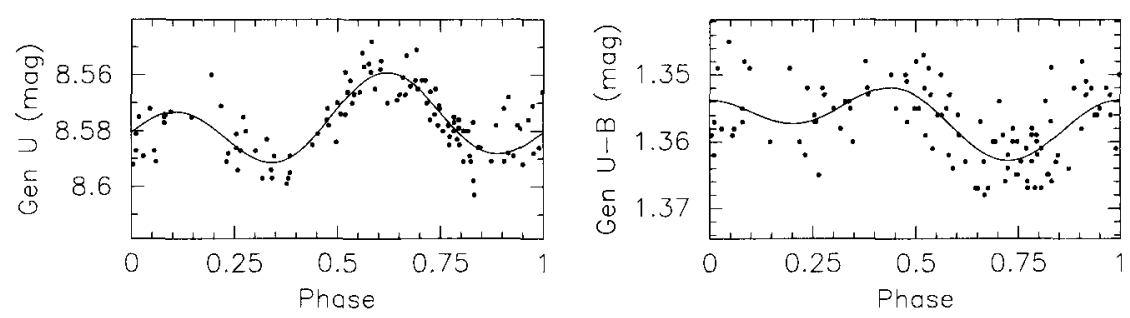

Figure 2. The observations of HD 154689 obtained with the Mercator telescope in the Geneva $U$ passband (left) and the Geneva $U-B$ colour (right) folded with the rotational frequency $\nu_{\text {rot }}=1.95792 \mathrm{~d}^{-1}$.
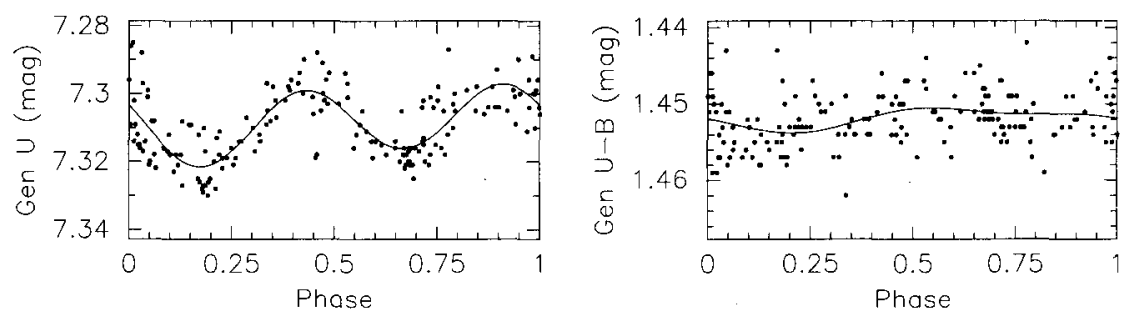

Figure 3. The observations of HD 112396 obtained with the Mercator telescope in the Geneva $U$ passband (left) and the Geneva $U-B$ colour (right) folded with the orbital frequency $\nu_{\text {orb }}=0.70727 \mathrm{~d}^{-1}$.

ima; main frequency not present in colors; e.g. HD 112396 in Fig. 3), or Be variations (classified as Be star; monoperiodic; observed frequency not compatible with expected theoretical frequency range). Their classification should be confirmed or ruled out by complementary high resolution spectroscopic observations. For two objects, no periodic variations are found. None of these other stars have been selected for long term monitoring with the Mercator telescope.

\section{Conclusions and future prospects}

We find that several of our target stars are multiperiodic variables. This result confirms that we are dealing with pulsating stars. These multiperiodic targets (full symbols in Fig. 1 and italic in Table 1) are selected for very long-term monitoring with the Mercator telescope.

\section{References}

Stellingwerf, R.F. 1978, ApJ, 224, 953

Scargle, J.D. 1982, ApJ, 263, 835

Pamyatnykh, A.A. 1999, Acta Astr., 49, 119 
Table 1. Overview of the results for the 28 studied B stars. For each target we give the HD number, the spectral type as given by the SIMBAD (CDS, Strasbourg, France), the number $N$ of Mercator observations, the total time-span $T$, and the frequencies found in the Mercator time series. Error estimates are given in brackets in units of the last decimals. The targets which were already classified as bonafide $\beta$ Cephei stars or SPB stars are indicated with respectively " $*$ " and "+". Italic frequencies indicate that we might have taken an alias frequency instead of a physical one because of a lack of extra information from the Hipparcos $H_{p}$ photometry or elsewhere. "..." denotes that there are clearly more intrinsic frequencies, but that it is impossible to choose frequency peaks due to the strong aliasing. Stars given in italic are selected for long-term monitoring.

\begin{tabular}{|c|c|c|c|c|c|c|}
\hline \multirow{2}{*}{\multicolumn{2}{|c|}{$\begin{array}{lr}\text { Star } \quad S p T \\
\beta \text { Cephei stars }\end{array}$}} & \multirow[t]{2}{*}{$N \quad T(\mathrm{~d})$} & \multicolumn{4}{|c|}{ frequencies $\left(d^{-1}\right)$} \\
\hline & & & \multirow{2}{*}{$\begin{array}{l}\nu_{1} \\
4.95555(5)\end{array}$} & \multirow{2}{*}{$\begin{array}{l}\nu_{2} \\
5.04829(3)\end{array}$} & \multirow{2}{*}{$\begin{array}{l}\nu_{3} \\
4.40338(5)\end{array}$} & \multirow{2}{*}{$\begin{array}{l}\nu_{4} \\
\ldots\end{array}$} \\
\hline HD 21803* & B2IV & 197377 & & & & \\
\hline$H D 203664 *$ & B0.5IIIn & $328 \quad 497$ & $6.02882(1)$ & $\ldots$ & & \\
\hline HD $163472 *$ & B2IV-V & 154426 & $7.14856(11)$ & & & \\
\hline HD $180642 *$ & B1.5II-III & 105419 & $5.48647(23)$ & & & \\
\hline HD $165174 *$ & BoIIIn & 149423 & $3.2854(11)$ & & & \\
\hline slowly pul & sating B & stars & $\nu_{1}$ & $\nu_{2}$ & $\nu_{3}$ & $\nu_{4}$ \\
\hline$H D 1976+$ & B5IV & 212511 & $0.39923(4)$ & $0.93895(8)$ & $0.79906(9)$ & $0.96630(9)$ \\
\hline HD 3379+ & $\mathrm{B} 2.5 \mathrm{IV}$ & 139417 & $1.82027(6)$ & $1.59388(35)$ & & \\
\hline$H D 21071+$ & $\mathrm{B} 7 \mathrm{~V}$ & 193504 & $1.18834(5)$ & $1.14919(11)$ & & \\
\hline$H D 28114$ & B6IV & 123411 & $0.48818(5)$ & $0.49275(9)$ & $0.63757(18)$ & \\
\hline$H D 179588$ & B9IV & 114473 & $0.85663(5)$ & $1.04031(3)$ & $1.19966(5)$ & \\
\hline$H D 182255+$ & B6III & 149472 . & $0.97186(5)$ & $0.79214(7)$ & $0.62521(3)$ & $\cdots$ \\
\hline HD 191295 & B7III & 168467 & $0.71911(7)$ & $\ldots$ & $\ldots$ & \\
\hline$H D 206540+$ & B5IV & 128507 & $0.72001(10)$ & $0.62098(7)$ & & \\
\hline$H D 208057+$ & $\mathrm{B} 3 \mathrm{Ve}$ & $\begin{array}{ll}76 & 467\end{array}$ & $0.89063(4)$ & $\ldots$ & & \\
\hline$H D 25558+$ & B3V & 108411 & $0.65259(7)$ & $\ldots$ & & \\
\hline$H D 28475$ & B5V & $45 \quad 382$ & $0.67447(7)$ & $\ldots$ & & \\
\hline$H D 222555$ & B9 & 215515 & $0.56413(8)$ & $\ldots$ & & \\
\hline HD 138003 & B9 & 315414 & $0.66110(6)$ & & & \\
\hline \multicolumn{3}{|c|}{ ellipsoidal variables? } & \multicolumn{4}{|l|}{$\nu_{\text {orb }}$} \\
\hline HD 24094 & B8 & $58 \quad 411$ & $0.95055(2)$ & & & \\
\hline HD 112396 & A0 & 174369 & $0.70727(7)$ & & & \\
\hline HD 149881 & B0.5III & 406418 & $0.19217(7)$ & & & \\
\hline HD 208727 & B8V & 296526 & $1.58389(5)$ & & & \\
\hline \multicolumn{3}{|c|}{ spotted stars? } & $\nu_{\text {rot }}$ & \multicolumn{3}{|l|}{$2 \nu_{\text {rot }}$} \\
\hline HD 46005 & $\mathrm{~B} 8 \mathrm{~V}$ & 168379 & $2.50474(13)$ & & & \\
\hline HD 154689 & B9 & 113127 & $1.95792(11)$ & $3.91584(11)$ & & \\
\hline HD 169820 & $\mathrm{~B} 9 \mathrm{~V}$ & 126147 & $2.12493(16)$ & & & \\
\hline Be star? & & & $\nu_{1}$ & & & \\
\hline HD 180968 & B0.5IV & 207472 & $0.63757(5)$ & & & \\
\hline constant $s$ & tars? & & & & & \\
\hline HD 13745 & $09.7 \mathrm{II}$ & 160377 & & & & \\
\hline HD 89688 & B3.2IV & 135289 & & & & \\
\hline
\end{tabular}

